PAGAMENTO DEL LATTE OVINO IN BASE ALLA QUALITÃ COME STRUMENTO PER I CONTROLLI PREVISTI DAI REGOLAMENTI COMUNITARI DI IGIENE: VALUTAZIONI CONSEGUENTI AL LIVELLO PRODUTTIVO AZIENDALE, TERRITORIO E STAGIONE DI PRODUZIONE

\title{
MILK PAYMENT SYSTEM AS A TOOL FOR THE APPLICATION OF THE EU RULES ON HYGIENE FOODSTUFFS: DIFFERENCES RELATED TO PRODUCTION LEVEL, PROVINCE AND SEASON OF PRODUCTION
}

\author{
Bozzi R. ${ }^{1}$, Dal Prà A. ${ }^{1}$, Brajon G. ${ }^{2}$ \\ ${ }^{1}$ Dipartimento di Scienze Zootecniche, Università degli Studi di Firenze - \\ ${ }^{2}$ Istituto Zooprofilattico Sperimentale delle Regioni Lazio e Toscana
}

SUMMARY

Key words

\begin{abstract}
The aim of the study was to evaluate a sheep milk payment of the milk produced in Tuscany in 2006 based on fat and protein levels. Data were collected twice a month in each of the 371 farms considered. Following the Tuscany Region recommendations three different levels of payment has been created. A neutral zone where the price of the milk remained invariant and other two levels, one positive (base milk price plus bonus) and one negative (base milk price minus bonus), both calculated by statistical procedures. The results showed that the period of the year markedly influenced the price paid and thus the payment grid has to be produced accordingly to these variations. Nonetheless the application of a payment system as the one proposed here needs a strong adjustment of the farms to the new quality requirements.
\end{abstract}

Milk payment, milk quality, sheep milk

\section{INTRODUZIONE}

L'applicazione dei Regolamenti Comunitari di igiene, prevede il monitoraggio della qualità igienico-sanitaria del latte nelle aziende di produzione. I controlli, sotto la diretta responsabilità degli allevatori, possono essere effettuati nell'ambito dei sistemi di pagamento differenziato in base alla qualità. Com'è noto, nel settore bovino le esperienze di pagamento in base alla qualità sono state numerose ed hanno portato ad un complessivo miglioramento della qualità del latte all'origine; per contro, nel set- tore ovino, tali esperienze sono poche e frammentate. Le principali difficoltà sono legate alla realizzazione di una rete di prelievi efficaci ed alla variabilità dei livelli produttivi che influenzano la qualità del latte nell'arco della lattazione. Volendo dunque utilizzare il pagamento in base alla qualità del latte di pecora come strumento per il monitoraggio dei parametri igienico-sanitari previsti dai Regolamenti Comunitari di igiene, è stata valutata l'influenza di alcuni fattori che determinano la variabilità dei parametri grasso e proteine che sono utilizzati come riferimento per la resa in formaggio. 


\section{MATERIALI E METODI}

Il data-set utilizzato per l'applicazione del modello proposto è stato ricavato da un monitoraggio effettuato nell'anno 2006 nella Regione Toscana. Sono state coinvolte 371 aziende agro-pastorali che allevano ovini di razza Sarda distribuite nelle 4 province con maggiore produzione di latte: Grosseto, Siena, Firenze e Pisa (Grafico 1). Il campionamento è stato effettuato da Gennaio a Luglio e sono stati prelevati secondo metodica standardizzata, con cadenza quindicinale, complessivamente 3670 campioni di latte di massa aziendale. I campioni sono stati inviati ai laboratori latte delle Sezioni di Firenze e Grosseto dell'Istituto Zooprofilattico e sono stati analizzati per: grasso, proteine, lattosio, residuo secco magro, cellule somatiche, indice crioscopico (Combyfoss, Foss Italia), carica batterica totale (Bacto-Scan 8000S, Foss Italia), e residui di antibiotici e sulfamidici (inibizione della crescita su piastra).

Grafico 1: Distribuzione delle aziende agropastorali per Provincia

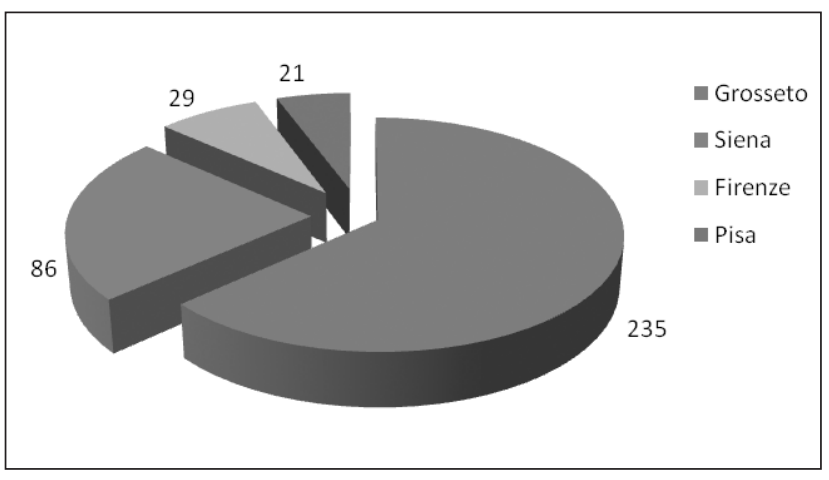

In ordine ai parametri chimici grasso e proteina, è stata inizialmente effettuata un'analisi della varianza per definire eventuali effetti influenti. $\mathrm{E}$ stata inoltre ipotizzata una griglia di pagamento basata su due punti neutri: uno per grasso "X" ed uno per proteine "Y". I punti neutri sono stati calcolati come media ponderata sui litri di latte per singola mungitura e sono stati suddivisi in base a tre stagioni di produzione: A) Gennaio-Marzo, B) AprileMaggio e C) Giugno-Luglio. E' stata definita una fascia neutra per il grasso, da "X"-0,3\% a "X" $+0,3 \%$ e per le proteine da Y"-0,2\% a "Y"+0,2\%. Ai campioni ricadenti nelle fasce neutre è stato applicato il prezzo concordato in sede regionale pari a 0,805 $€ /$ litro. Per ogni incremento di grasso pari a $0,2 \%$, rispetto al valore di $\mathrm{X}+0,3 \%$, è stato concesso un premio di 0,005 €/litro, fino ad un massimo di + $0,026 € /$ litro, per ogni decremento di grasso pari a $0,2 \%$ rispetto al valore di X $-0,3 \%$ è stata applicata una detrazione di 0,005 €/litro, fino ad un massimo di $-0,026 € /$ litro. Per quanto riguarda le proteine, per ogni incremento pari a $0,2 \%$ in più, rispetto al valore di $\mathrm{Y}+0,2 \%$, è stato concesso un premio di 0,005 $€ /$ litro, fino ad un massimo di $+0,026 € /$ litro, per ogni decremento pari a $0,2 \%$ in meno rispetto al valore di Y $-0,2 \%$ è stata applicata una detrazione di $0,005 € /$ litro, fino ad un massimo di -0,026 €/litro.

Successivamente è stato calcolato, per ogni campione prelevato da ciascun tank refrigerante il prezzo per litro ponderato per il numero di munte presenti nel tank stesso. I dati sono stati sottoposti ad analisi della varianza impiegando la procedura GLM del pacchetto statistico SAS $^{\circledR}$ (2003), considerando come effetti fissi: periodo di produzione: A) Gennaio-Marzo, B) Aprile-Maggio e C) Giugno-Luglio, provincia (Siena, Grosseto, Firenze e Pisa) e livello produttivo aziendale $(1=0-25,2=25-50,3=50$ 100, $4=100-200,5=>200$ ) espresso come litri per mungitura. E' stata inoltre valutata l'interazione dei fattori provincia/periodo di prelievo.

\section{RISULTATI}

In tabella 1 sono riportate le medie ponderate per i parametri grasso e proteina suddivise nei tre differenti periodi di prelievo.

Tabella 1: Medie ponderate di proteine e grasso per periodo di prelievo

\begin{tabular}{lccc}
\hline Periodo & $\begin{array}{c}\text { Numero } \\
\text { osservazioni }\end{array}$ & Grasso “X” & Proteine "Y” \\
\hline A) Gennaio-Marzo & 1504 & $6,15 \%$ & $5,49 \%$ \\
\hline B) Aprile-Maggio & 1300 & $5,97 \%$ & $5,54 \%$ \\
\hline C) Giugno-Luglio & 866 & $6,73 \%$ & $5,64 \%$ \\
\hline
\end{tabular}

La distribuzione dei campioni analizzati secondo il sistema proposto, rapportata ai 3 periodi di raccolta, è rappresentata per il grasso nel grafico 2 e per le proteine nel grafico 3.

L'analisi degli effetti fissi ha evidenziato come significativamente importanti: azienda, numero di mungiture (solo per il parametro proteine), stagione di prelievo e covariata del numero dei litri presenti nel tank refrigerante al momento del prelievo (tabella 2). 
Grafico 2: Distribuzione dei campioni in relazione alle diverse fasce di premio o penalizzazione per il grasso

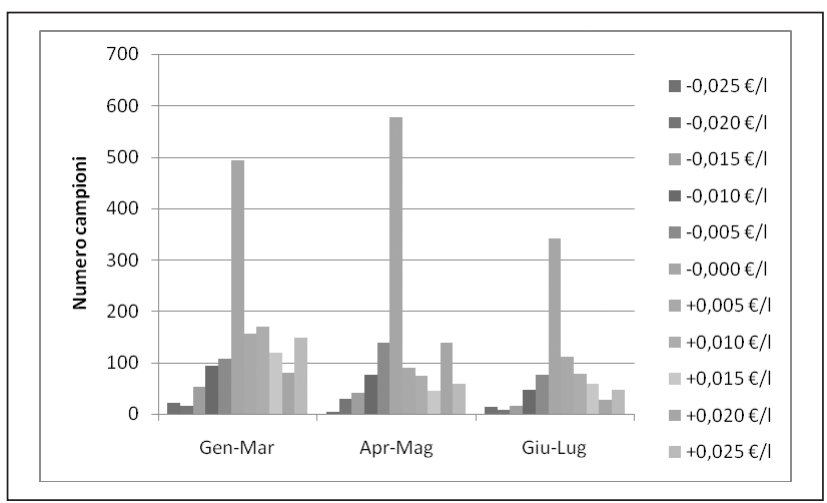

Grafico 3: Distribuzione dei campioni in relazione alle diverse fasce di premio o penalizzazione per le proteine

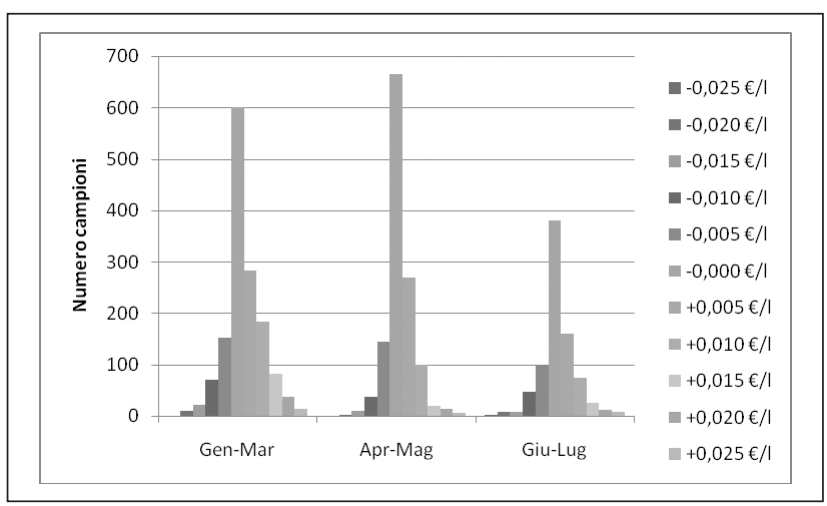

Tabella 2: Significatività degli effetti fissi

\begin{tabular}{lcc}
\hline Fonte di variazione & Grasso \% & Proteine \% \\
\hline Azienda & $<0,0001$ & $<0,0001$ \\
\hline Numero munte & 0,2404 & $<0,0001$ \\
\hline Stagione & $<0,0001$ & $<0,0001$ \\
\hline Litri (covariata) & $<0,0001$ & 0,0005 \\
\hline
\end{tabular}

Nel grafico 4, risulta evidente come il prezzo del latte tenda ad aumentare al diminuire del livello produttivo aziendale: nella categoria 0-25 litri, la meno produttiva, il prezzo è pari a $0,809 € /$ litro mentre nella categoria oltre 200 litri, la più produttiva, il prezzo è pari a $0,793 € /$ litro.

In relazione al periodo di produzione, nel periodo $\mathrm{A}$ (Gennaio-Marzo) il prezzo più elevato è pagato in
Grafico 4: Distribuzione del prezzo per litro in relazione al livello produttivo aziendale

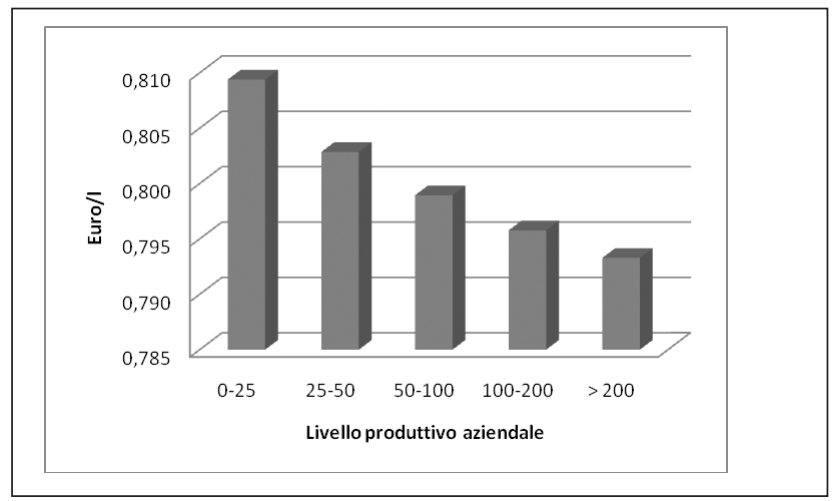

provincia di Pisa $(0,802 € /$ litro), nei periodi $\mathrm{B}$ (Aprile-Maggio) e C (Giugno-Luglio) in provincia di Siena (rispettivamente $0,803 € / /$ itro e $0,802 € /$ litro). Per contro, i prezzi più bassi sono pagati nei periodi A (Gennaio-Marzo) e B (Aprile-Maggio) in provincia di Firenze (rispettivamente 0,795 €/litro e 0,801 $€ /$ litro) e nel periodo C (Giugno-Luglio) in provincia di Pisa $(0,795 € /$ litro). Le differenze non sono di entità elevata, vista la numerosità del campione, tuttavia sono fra loro significative ed evidenziano l'influenza del fattore legato all'anticipazione della stagione di lattazione nelle province costiere (Grosseto e Pisa).

Grafico 5: Distribuzione dei campioni in relazione a prezzo per litro, province e periodo di produzione

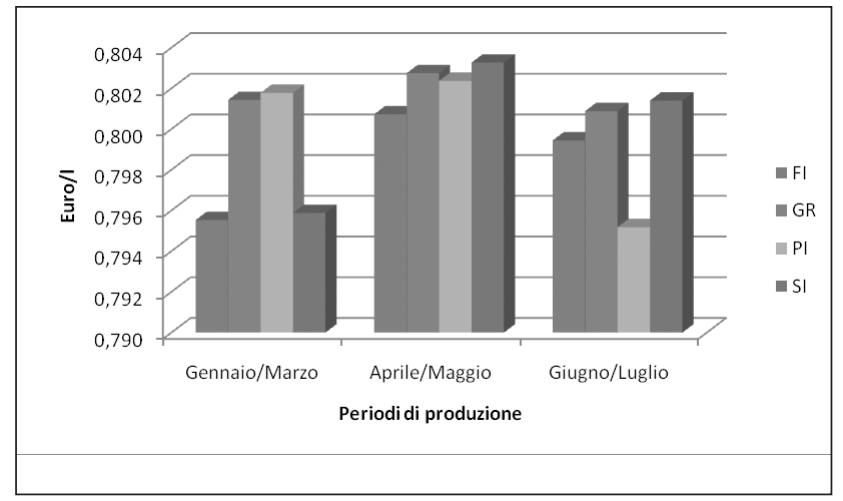

Nell'interazione tra periodo di produzione e livello produttivo aziendale i prezzi più elevati sono pagati nelle categorie con i minori livelli produttivi aziendali soprattutto nei periodi A) e C) mentre tendono a livellarsi nella stagione intermedia B). 


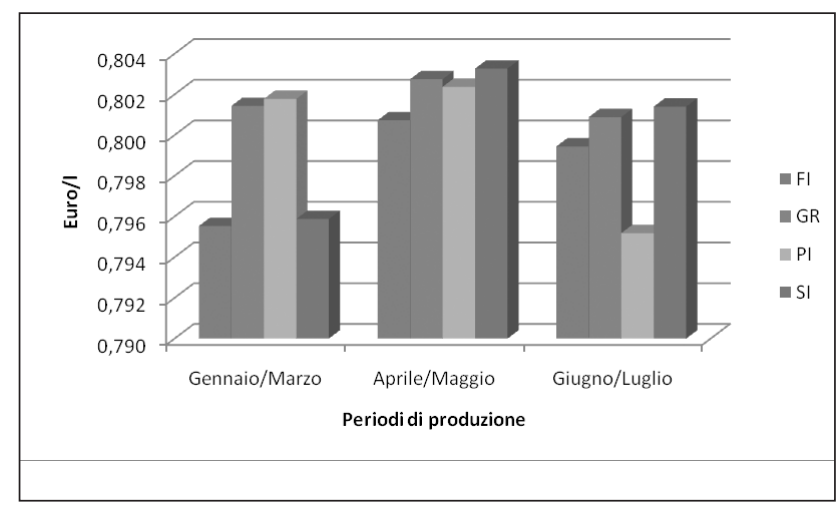

\section{CONCLUSIONI}

Il pagamento differenziato del latte in base a parametri qualitativi gioca un ruolo positivo sul miglioramento delle caratteristiche chimico-fisiche del latte all'origine. In un tale sistema gli allevatori tendono infatti a considerare importante non solo la quantità prodotta ma anche la qualità; al tempo stesso un controllo continuo del prodotto latte, necessario per la definizione del pagamento, porta anche a porre maggiore attenzione alle caratteristiche igienico-sanitarie del latte stesso con indubbi vantaggi in tal senso. Nel settore ovino uno strumento simile deve prevedere l'organizzazione di una rete di prelievo dei campioni particolarmente efficace. Alcune valutazioni devono inoltre essere fatte per le differenze legate alle diverse zone di produzione, alla stagione, ai livelli produttivi aziendali ed alle razze allevate. Nel monitoraggio condotto in Toscana nell'anno 2006, su 371 aziende che allevano pecore di razza Sarda, applicando una griglia sperimentale per grasso e proteine, è stato confermato come i sopraddetti fattori potrebbero influenzare l'avvio del sistema di pagamento differenziato in base alla qualità; in particolare sarà necessario disporre di diverse griglie in relazione al periodo di produzione. È risultato inoltre che le aziende produttrici della maggiore quantità di latte risulterebbero in una prima fase di applicazione del sistema penalizzate; d'altra parte la selezione genetica fino ad oggi effettuata nel settore della pecora da latte si è basata solo sulla quantità e non sulla qualità. Resta comunque vera l'affermazione che un'attivazione del sistema di pagamento del latte ovino in base alla qualità, rappresenta uno strumento utile per implementare il monitoraggio dei parametri igienico sanitari previsti dall'applicazione dei regolamenti comunitari di igiene; il sistema trova tuttavia alcune difficoltà di avvio sia per la complessità della rete dei campionamenti sia per l'iniziale diffidenza degli allevatori.

\section{BIBLIOGRAFIA}

1) Barrel G.K. (1989). Milk payment and quality. Animal Industries Workshop, May 1989. Christchurch (New Zealand). Lincoln College, 9-11.

2) Brajon G., Colafrancesco R. (2006). Caratterizzazione del latte ovino in funzione dell'ottimizzazione della resa in formaggio. La razza Sarda in Toscana. L'allevatore di ovini e caprini, 1, 6-7.

3) Brajon G., Olivetti A., Benedetti M., Casini M., Brizioli N.R. (2004). Ewes milk quality in Tuscany in relation to compositional payment system. International Symposium on Dairy Safety and Hygiene, 25 March 2004, Cape Town, South Africa.

4) Brajon G., Gradassi S. (2006). Ipotesi per l'avvio di un sistema di pagamento differenziato del latte ovino in base alla qualità in Toscana. L'allevatore di ovini e caprini, $2,3-5$.

5) Suejan L. (2006). The study on the feasibility of using milk composition to substitute milk specific gravity to fit into raw milk payment system. Journal of Taiwan Livestock Research, 39, 3, 165-173.

6) Nyborg N.J. (1998). The milk payment system in Denmark. Scandinavian Dairy Information, 12, 3, 38-39. 